\title{
Can I Recognize My Body's Weight? The Influence of Shape and Texture on the Perception of Self
}

\author{
IVELINA V. PIRYANKOVA, Max Planck Institute for Biological Cybernetics \\ JEANINE K. STEFANUCCI, University of Utah \\ JAVIER ROMERO, Max Planck Institute for Intelligent Systems \\ STEPHAN DE LA ROSA, Max Planck Institute for Biological Cybernetics \\ MICHAEL J. BLACK, Max Planck Institute for Intelligent Systems \\ BETTY J. MOHLER, Max Planck Institute for Biological Cybernetics
}

The goal of this research was to investigate women's sensitivity to changes in their perceived weight by altering the body mass index (BMI) of the participants' personalized avatars displayed on a large-screen immersive display. We created the personalized avatars with a full-body 3D scanner that records the participants' body geometry and texture. We altered the weight of the personalized avatars to produce changes in BMI while keeping height, arm length, and inseam fixed and exploited the correlation between body geometry and anthropometric measurements encapsulated in a statistical body shape model created from thousands of body scans. In a $2 \times 2$ psychophysical experiment, we investigated the relative importance of visual cues, namely shape (own shape vs. an average female body shape with equivalent height and BMI to the participant) and texture (own photorealistic texture or checkerboard pattern texture) on the ability to accurately perceive own current body weight (by asking the participant, "Is it the same weight as you?"). Our results indicate that shape (where height and BMI are fixed) had little effect on the perception of body weight. Interestingly, the participants perceived their body weight veridically when they saw their own photo-realistic texture. As compared to avatars with photo-realistic texture, the avatars with checkerboard texture needed to be significantly thinner in order to represent the participants' current weight. This suggests that in general the avatars with checkerboard texture appeared bigger. The range that the participants accepted as their own current weight was approximately a $0.83 \%$ to $-6.05 \%$ BMI change tolerance range around their perceived weight. Both the shape and the texture had an effect on the reported similarity of the body parts and the whole avatar to the participant's body. This work has implications for new measures for patients with body image disorders, as well as researchers interested in creating personalized avatars for games, training applications, or virtual reality.

Categories and Subject Descriptors: I.3.m [Computer Graphics]: Miscellaneous

General Terms: Three-Dimensional Graphics and Realism, Vision and Scene Understanding

Additional Key Words and Phrases: Body perception, virtual environments, human perception and performance, visual psychophysics, texture, 3D shape, BMI, avatar

ACM Reference Format:

Ivelina V. Piryankova, Jeanine K. Stefanucci, Javier Romero, Stephan De La Rosa, Michael J. Black, and Betty J. Mohler. 2014. Can I recognize my body's weight? The influence of shape and texture on the perception of self. ACM Trans. Appl. Percept. 11, 3, Article 13 (September 2014), 18 pages.

DOI: http://dx.doi.org/10.1145/2641568

Authors' address: email: ivelina.piryankova@tuebingen.mpg.de.

Permission to make digital or hard copies of part or all of this work for personal or classroom use is granted without fee provided that copies are not made or distributed for profit or commercial advantage and that copies show this notice on the first page or initial screen of a display along with the full citation. Copyrights for components of this work owned by others than ACM must be honored. Abstracting with credit is permitted. To copy otherwise, to republish, to post on servers, to redistribute to lists, or to use any component of this work in other works requires prior specific permission and/or a fee. Permissions may be requested from Publications Dept., ACM, Inc., 2 Penn Plaza, Suite 701, New York, NY 10121-0701 USA, fax +1 (212) 869-0481, or permissions@acm.org.

(C) 2014 ACM 1544-3558/2014/09-ART13 $\$ 15.00$

DOI: http://dx.doi.org/10.1145/2641568 


\section{INTRODUCTION}

Human bodies are possibly the most familiar objects that people encounter daily. The most familiar of all bodies is likely one's own body, as it is observed every day [Longo and Haggard 2012b]. The visual perception of one's own body is an aspect of body image. Body image is the phrase used to describe the mental, multimodal perceptual, conceptual, or emotional representation of one's body, which involves sensory inputs perceived through first-person experience and through the experience of the body as an object in the physical world [De Vignemont 2011; Schilder 1935; Riva 2010, 2011; Piryankova et al. 2014].

Recent research suggests that even healthy participants perceive a mismatch between their actual body size and their body image, albeit not as much as patients with eating disorders [Mischner et al. 2013; Hashimoto and Iriki 2013; Longo and Haggard 2012a]. The precision of one's own body size perception can vary due to many factors [Grogan 2007], such as psychological condition, thoughts about ideal body size, exposure to media [Cash et al. 1983; Mischner et al. 2013], and body satisfaction [Mischner et al. 2013]. Hashimoto and Iriki [2013] used distorted photographs of participants' own bodies to show that women estimate photographs distorted by $10 \%$ in width to be photographs of their current body size, but photographs distorted by $30 \%$ in width were estimated to belong to others. Other work finds that healthy individuals show large distortions in size and shape of their hand [Longo and Haggard 2012a] as well as their body [Fuentes et al. 2013], suggesting that people perceive both their hand and body to be wider and shorter compared to their actual size. Mischner et al. [2013] showed that exposure to music videos can bias both the perceived and the ideal body size of healthy women. Likewise, a large contrast between the idealized body promoted by the popular media (TV, music videos) and the average woman's body size [Mischner et al. 2013] contributes to lower self-esteem and negative self-perceived attractiveness in healthy women [Cash et al. 1983; Mischner et al. 2013]. The discrepancy between women's perceived and ideal body size has been used to detect body dissatisfaction and bulimic tendencies [Cash and Deagle 1997], because people with eating disorders do not veridically perceive their own body size [Glucksman and Jules 1968; Cash and Deagle 1997; Smeets et al. 1997] (see Farrell et al. [2005] for an overview). More specifically, patients who are anorexic tend to overestimate the size of their body [McCabe et al. 2001], whereas patients who are obese underestimate both their own and the body size of others [Glucksman and Jules 1968; Doolen et al. 2009].

Body image has been assessed in many experiments using a variety of body size measures (see Grogan [2007] for an overview of the literature), such as figure rating scales [Grogan 2007; Fallon and Rozin 1985; Stunckard et al. 1983], drawing one's body [Askevold 1975], behavior matching, and affordance measures [Slade and Russell 1973] (see Farrell et al. [2005] for an overview). Most studies assessing body perception have used optical techniques to manipulate body shape, such as distorted videos [Freeman et al. 1984; Brodie et al. 1989], distorted mirrors [Traub and Orbach 1964], or distorted photographs [Hashimoto and Iriki 2013]. However, these methods usually apply uniform width changes to the visually portrayed body geometry and thus result in unrealistic body deformations. However, in reality, gaining weight does not result in uniform changes in body geometry but rather in multidimensional nonuniform changes. For this reason, when measuring body size perception, some researchers have used figure rating scales [Grogan 2007; Fallon and Rozin 1985; Stunckard et al. 1983; Longo and Haggard 2012a] that considered shape changes and fat distribution. Still, it is often difficult for the individual to identify with a figure not depicting features specific to the person, such as shape particularities, wrinkles, skin color, freckles, and moles. Each of these features may impact the precision of body perception. Further, this might be a potential reason why several studies show that estimations of the size of the whole body produce more accurate results than estimates of separate body parts [Cash and Deagle 1997; Smeets et al. 1997]. 

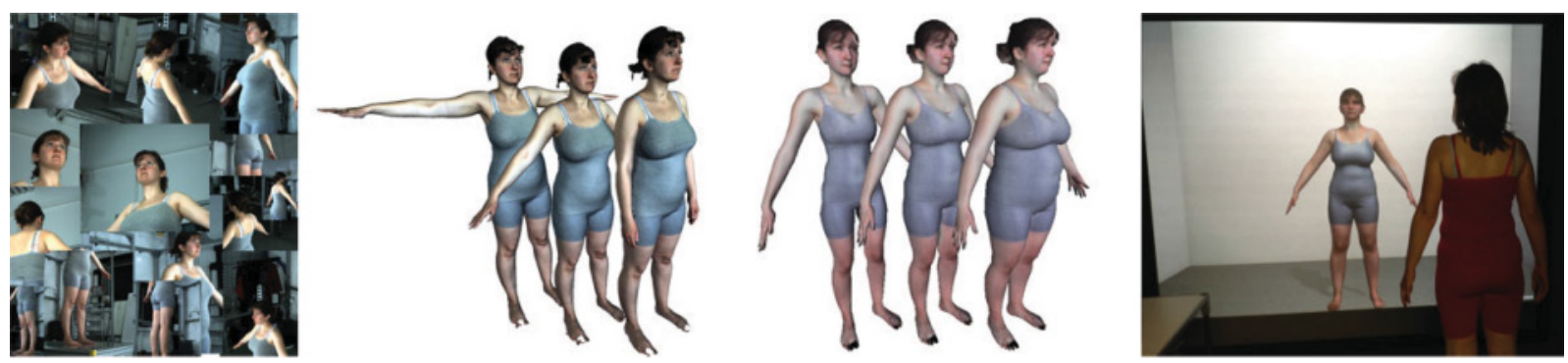

Fig. 1. (From left to right) First, we captured each participant's body geometry and image texture using a 3D scanning system. Second, we captured three poses (T-pose, A-pose, and natural pose), resulting in high-resolution 3D meshes. Third, we fit a statistical model of body shape and pose variation to the scans, bringing them into correspondence; using this model, we created a set of personalized avatars for each subject with varying BMIs (ranging from $\pm 20 \%$ BMI change). Fourth, we assessed women's sensitivity to changes in their perceived weight, asking them to estimate whether the size of the participants' personalized avatars displayed on a large-screen immersive display is the same as their current weight.

Although considerable literature has examined how shape from shading is involved in object perception (see Schofield et al. [2013]), fewer studies have looked at the influence of color information (i.e., changes in clothing or in texture information) on human body perception. Thompson and Mikellidou [2011] showed an effect of texture pattern (horizontal or vertical lines) on the perception of the body size of a pictured mannequin. The effect of the texture pattern is consistent with the Helmholtz visual illusion, in which the length of the vertical stripes need to be extended vertically to match the perceived extent of the horizontal stripes, whereas horizontal stripes need to be increased in width to match the perceived length of the vertical stripes. Their results are in direct opposition to the wellknown fashion tip that clothes with horizontal patterns make you look fatter but vertical stripes are slimming. To our knowledge, no study has yet investigated both shape and texture information for the perception of human body image, possibly due to the difficulty in implementing these changes with optical techniques.

The goal of this study is to investigate the accuracy of women's perception of their own body weight. We explore this by altering the body mass index (BMI) of the participants' personalized avatars displayed on a large-screen immersive stereoscopic display and additionally by manipulating two visual cues: shape and texture. To create a personalized avatar for each participant, both the participant's body geometry and texture were recorded with a full-body 3D scanner (Figure 1). The 3D scans were first fit with a body model that decomposed body deformations into identity and pose [Hirshberg et al. 2012] and created a color model of the person [Bogo et al. 2014]. The correlation between identity deformations and anthropometric measurements observed in training data, encapsulated in a statistical body shape model created from thousands of body scans, allowed us to apply deformations to the avatars according to specific measurement changes (i.e., changes in body height, BMI, and specific body part lengths [Weiss et al. 2011]). Thus, we used a novel approach to assess body perception by altering the weight of personalized avatars to produce changes in BMI $(0 \%, \pm 5 \%, \pm 10 \%, \pm 15 \%, \pm 20 \%$ change in current BMI) while keeping other body size parameters fixed (i.e., height, arm length, inseam). Further, the personalized avatars allowed us to assess the influence of the visual cues on the accuracy of perceived body weight. We investigated the relative importance of two visual cues, namely shape (own shape vs. an average female body shape with equivalent height and BMI to the participant) and texture (own photo-realistic texture or a checkerboard patterned texture) on the ability to accurately perceive own body weight. The experiment employed a 2 (shape: own and average) $\times 2$ (texture: own photo-realistic and checkerboard) design, in which we assessed participants' sensitivity to their own body weight using one-interval forced choice with two response alternatives (2AFC) psychophysical 
Table I. Descriptive Statistics of the Participants $(N=13)$

\begin{tabular}{|c|c|c|c|}
\hline & Range-Min. & Range-Max. & Mean \\
\hline BMI & 16.70 & 27.30 & $21.75(S D=2.92)$ \\
\hline Height (m) & 1.54 & 1.78 & $1.67(S D=.06)$ \\
\hline Current weight (kg) & 49.00 & 74.00 & $60.68(S D=7.09)$ \\
\hline $\begin{array}{l}\text { Self-esteem (15-25 normal range; smaller than } \\
15 \text { indicates lower self-esteem, greater than } 25 \\
\text { indicates higher self-esteem) }\end{array}$ & 11.00 & 28.00 & $21.46(S D=5.44)$ \\
\hline Age (y) & 20.00 & 37.00 & $27.38(S D=4.48)$ \\
\hline Self-reported ideal weight $(\mathrm{kg})$ & 45.00 & 67.00 & $57.69(S D=6.14)$ \\
\hline Self-reported lowest weight $(\mathrm{kg})$ & 48.00 & 65.00 & $54.23(S D=5.83)$ \\
\hline Self-reported highest weight $(\mathrm{kg})$ & 49.00 & 80.00 & $64.00(S D=8.45)$ \\
\hline $\begin{array}{l}\text { Height/weight distance (see Section } 3.3 \text { for more } \\
\text { information) }\end{array}$ & 5.91 & 0.16 & $1.29(S D=1.59)$ \\
\hline $\begin{array}{l}\text { Selected measurements distance (see Section } 3.3 \\
\text { for more information) }\end{array}$ & 3.54 & 15.94 & $7.72(S D=3.93)$ \\
\hline
\end{tabular}

procedure, where the participants were asked, "Is it the same weight as you?" Our aim was to find the range of body sizes that healthy individuals perceive as their current weight. At the end of the 2AFC trials, we also asked participants to adjust the size of the avatar to match their current and ideal weight using a method of adjustment task, which enabled the participant to set the intensity of the visual stimuli. We hypothesized that the current weight adjustment would be consistent with the $2 \mathrm{AFC}$ results, but the ideal weight was assessed to provide us with an indirect measure of body weight satisfaction. Finally, the participants were asked to rate the level of similarity between the body of the avatar and their own body. Our findings may contribute to the design of tasks in which visual stimuli that consider own shape and identity, as provided through a photo-realistic texture, may be important (i.e., body image assessment tasks for patients with body image disorder).

\section{METHOD}

\subsection{Participants}

In our study, 13 female participants who had no history of eating disorders were compensated with eight Euro per hour for their participation (Table I). Informed written consent was obtained prior to body capture and the experiment. Participants and the data were treated according to the Declaration of Helsinki. The recording methods of the database and the subsequent validation experiment were approved by the local ethics committee of the Eberhard Karls University of Tübingen.

\subsection{Technical Setup: Generating the Visual Stimuli}

Body scans from the participants were collected using a 3D full-body scanning system (3dMD, Atlanta, GA). The system is composed of 22 stereo units, each including a pair of black and white cameras observing the textured light pattern projected by speckle projectors, and a 5-megapixel color camera that captures the body texture. The system provides very good coverage of the human body in different poses and can resolve the $3 \mathrm{D}$ locations of a point on the body to approximately $1 \mathrm{~mm}$. Each participant was scanned in three poses (A-pose, T-pose, and neutral) (see Figure 1, second to the left).

First, body scans of our participants were captured and then we registered a 3D body model to the participants' scans. Through this process, we obtained 3D meshes and textures that resembled the original 3D scans but that shared a common topology, which allowed us to draw statistical conclusions 
from them. For the scan registration, we use a statistical body model that compactly parametrizes body deformations in terms of deformations due to subjects' identity and body pose [Hirshberg et al. 2012; Anguelov et al. 2005]. Once registered, we extracted a texture map per scan after decomposing the colors observed in the RGB images into albedo and irradiance [Bogo et al. 2014]. The final avatar texture map was computed as the median of each individual scan map.

Our statistical body model is learned from registered 3D scans of nearly 2,000 different bodies in a single pose and thousands of 3D scans of many people in many different poses. From the registered scans of different people, we learn a low-dimensional statistical model of body shape variation in the population. Following Anguelov et al. [2005], we compute the deformation of a triangulated template mesh to every scan. Each triangle is described by a $3 \times 3$ deformation matrix that captures how the triangle has to deform to match the scan. An individual body shape is then characterized by a vector containing all of the deformations that transform the template into the scan; this is a very high-dimensional description of body shape. To capture the correlated shape statistics in the population, we compute these deformations for 2,094 female bodies in the CAESAR dataset [Robinette and Daanen 1999]. We then perform principal component analysis on the deformations and model body shape variation in a subspace, $U$, spanned by the first 300 principal components. This allows us to compactly describe the shape of an individual, $S_{j}$, in terms of a vector of 300 linear coefficients, $\beta_{j}$, that approximate the shape deformations as $S_{j}=U_{j} \beta_{j}+\mu$, where $\mu$ is the mean shape deformation in the population. Here, we model only female body shape.

To modify body shape, while keeping some anthropometric measurements fixed, we must model the relationship between identity deformation coefficients and relevant measurements. Similar to Weiss et al. [2011], we model the relation between identity deformations $\beta$ and anthropometric measurements $A$ as a linear mapping. Since it is linear, this mapping also models the relation between changes in shape coefficients and measurements, which will allow us to modify $\beta$ in a way that produces the intended measurement changes.

Mathematically, learning the linear relation between $A$ and $\beta$ corresponds to a least squares problem $(A \mid 1) X=\beta$, where $A=\left[A_{1} \ldots A_{n}\right]$ is a matrix of size $n \times m$ containing the $m=4$ measurements of $n=2,094$ CAESAR bodies, $\beta=\left[\beta_{1} \ldots \beta_{n}\right]$ is a matrix $n \times b$ containing the $b=300$ identity deformation coefficients, and $X$ is a matrix $(m+1) \times b$ to be estimated. $X$ describes the mapping between deformation coefficients $\beta$ and the measurements $\left[w, h, a, i, b_{0}\right]$ (weight, height, arm length, inseam, and deformation intercept). However, it also describes the relation between shape and measurement changes; for a given subject with shape coefficients $\beta_{i}$ and measurements $A_{i}$ (so that $A_{i} X=\beta_{i}$ ), changes in measurements $\Delta_{A}$ correspond to changes in deformation coefficients $\Delta_{\beta}=\Delta_{A} X$, since $\left(A_{i}+\Delta_{A}\right) X=\beta_{i}+\left(\Delta_{A} X\right)$. An example of these shape changes applied to an average body can be visualized at http://bodyvisualizer.com/ [Perceiving Systems MPI IS 2011].

Given each subject's weight $w$, height $h$, and 3D registration, the personalized avatars were constructed with nine varying BMIs $\left(1+\frac{\Delta b m i}{100}\right) \frac{w}{h^{2}}$, where $\Delta_{b m i}=\{0, \pm 5, \pm 10, \pm 15, \pm 20\}$. This was achieved by changing their identity deformation coefficients by $\Delta_{\beta}=\left[\frac{\Delta_{b m i}}{100} w, 0,0,0,0\right] \cdot X$ (i.e., applying a change in weight equal to the desired proportional change in BMI while keeping the rest of the measurements constant).

Additionally, another avatar was created with same height $h$ and weight $w$ as the participant but with a different overall shape (e.g., different fat distribution, limb lengths). To achieve this, we first chose the individual (with height and weight, $h_{\text {avg }}, w_{\text {avg }}$ ) in the CAESAR dataset whose shape and body morphology was closest to the average female shape. Then, we made the height and weight of this individual's avatar match the participant's nine varying BMIs previously computed by changing the deformation coefficients by $\Delta_{\beta}=\left[\frac{\Delta_{b m i}}{100} w+\left(w-w_{\text {avg }}\right),\left(h-h_{\text {avg }}\right), 0,0,0\right] \cdot X$. 
(A)
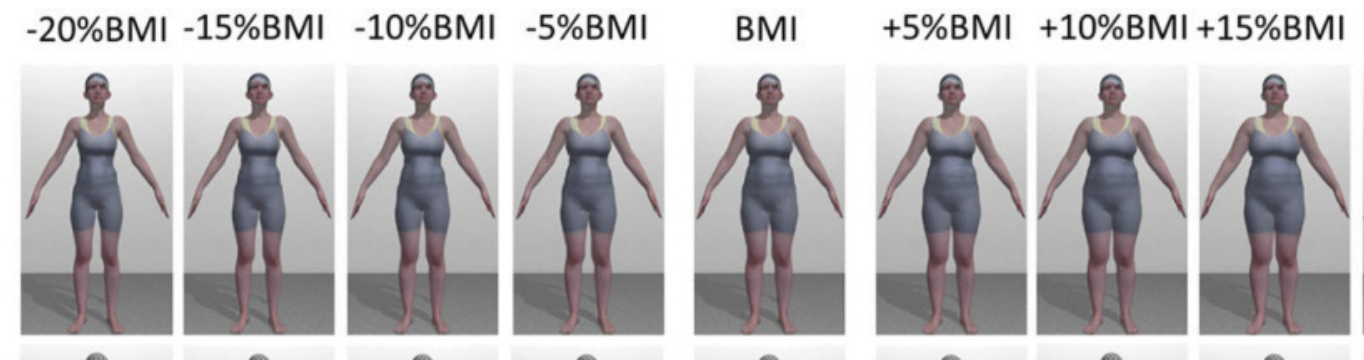

$+20 \% \mathrm{BMI}$

(B)
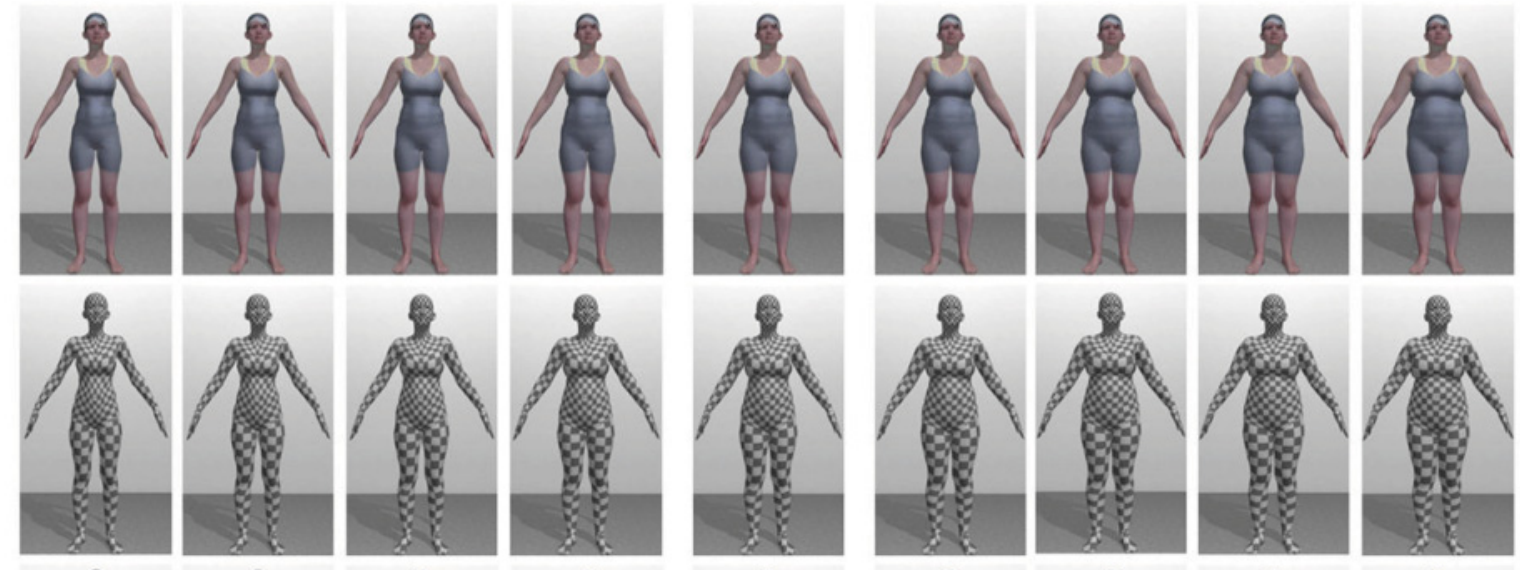

(C)
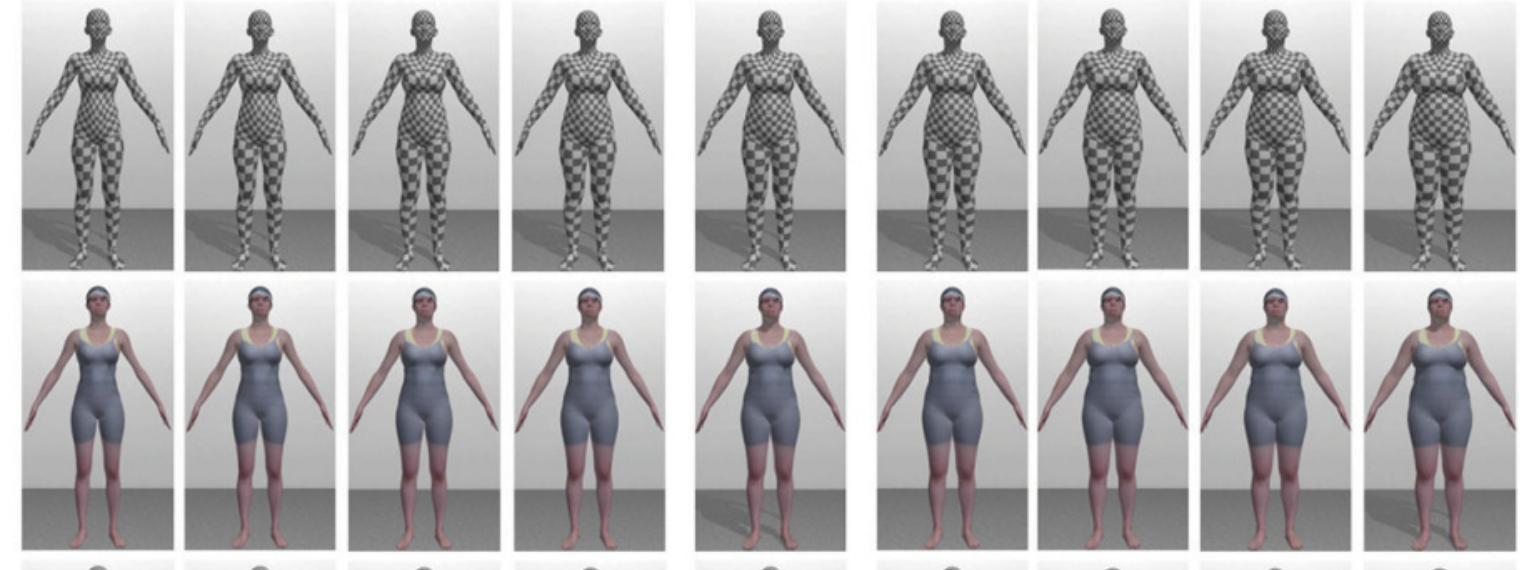

(D)
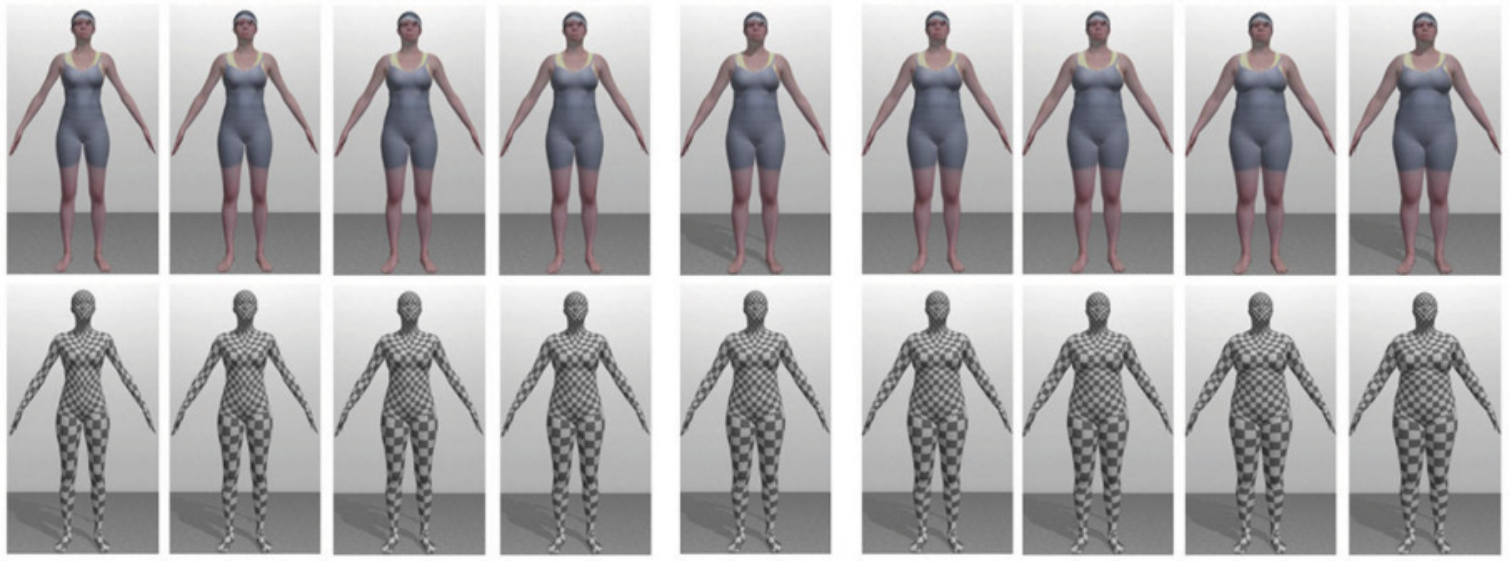

Fig. 2. Picture with all meshes in the A-pose for one participant. Row (A) shows the set of visual stimuli representing an avatar with own photo-realistic texture and own shape. Row (B) shows the set of visual stimuli representing an avatar with checkerboard pattern texture and own shape. Row (C) shows the set of visual stimuli representing an avatar with own photorealistic texture and average shape. Row (D) shows the set of visual stimuli representing an avatar with checkerboard pattern texture and average shape. Notice, for example, that shape deformations in the avatar apply to all body parts in a nonuniform way, as can be noticed in this $2 \mathrm{D}$ image in the ears, legs, and the stomach area, especially when comparing the lowest BMI to the greatest BMI avatar.

Thus, the nine variations of the average body had different relative body proportions and shape than that of the participant but the same height and BMI as the nine meshes generated from the participant's anthropomorphic data (Figure 2). The texture mapping and mesh topology were the same for all meshes. The texture used was either the photo-realistic texture previously described or a checkerboard texture that removes color details specific to the subject.

For each participant, we combined the nine meshes generated by the $3 \mathrm{D}$ scanning system in a morphable mesh in Autodesk 3ds Max. We also created a morphable mesh for the nine average meshes with varying BMIs. The photo-realistic texture of each participant was postprocessed in Adobe Photoshop to match the color differences due to lighting between the participants' textures. Then, the participant's photo-realistic texture was directly applied to both the participant's shape meshes and 
the average shape meshes. Unity 3D was used for programming the experiment and setting the scene in which the mesh of the personalized and average avatar with either texture were loaded.

\subsection{Visual Stimuli: Virtual Scene}

The virtual scene included an empty virtual room with a personalized avatar. Prior work has shown that there are orientation-dependent recognition effects in object and shape perception [Bülthoff and Edelman 1992], as well as face perception [Wallraven et al. 2002]. Recent research also suggests that there may be orientation-dependent recognition effects in the perception of body size [Hashimoto and Iriki 2013]. Thus, the personalized avatar was shown in a constant A-pose from a frontal view, as if the participant were looking at herself in the mirror (a familiar and natural perspective). The personalized avatar was placed at a distance of $2 \mathrm{~m}$ in front of the participant.

\subsection{Technical Setup: Projecting the Visual Stimuli}

The visual stimuli were projected on a flat, large-screen immersive display (flat LSID) using a Christie $\mathrm{SX}+$ stereoscopic video projector with a resolution of $1,400 \times 1,050$ pixels. The projection surface covered an area of $2.16 \times 1.62 \mathrm{~m}$. The projection surface enabled a field of view (FOV) of 94.4 degrees horizontal and 78 degrees vertical at a viewing distance of $1 \mathrm{~m}$. The projection plane of the flat LSID was located at $0.265 \mathrm{~m}$ above the real floor. The flat LSID was connected to a ART@ SMARTTRACK motion tracking system, which included two tracking cameras and one rigid object with reflective markers. Our stereoscopic projection was generated using the average interpupillary distance (IPD), which is $0.065 \mathrm{~m}$ [Willemsen et al. 2008]. To see the virtual scene stereoscopically, the participant had to wear NVIDIAC shutter glasses. The design of the shutter glasses was similar to that of regular glasses. The shutter glasses had a horizontal FOV of 103 degrees and vertical FOV of 62 degrees, which covered an area of about $2.52 \times 1.2 \mathrm{~m}$ of the flat LSID when viewed from a distance of $1 \mathrm{~m}$.

\subsection{Experimental Design}

We measured participants' sensitivity to weight changes of their personalized avatar's body (measured in terms of BMI) and manipulated the body's texture (own vs. checkerboard) and shape (own vs. average) in one interval with $2 \mathrm{AFC}$ and in a method of adjustment task. Specifically, participants' task in the 2AFC part was to report whether the current presented avatar had the same body weight as themselves. To this end, we had a 2 (visual texture: own vs. checkerboard) $\times 2$ (shape: own vs. average) $\times 9$ (avatar BMI: $0 \%, \pm 5 \%, \pm 10 \%, \pm 15 \%, \pm 20 \%$ of current participant's BMI) completely crossed, within-participants design (see Figure 2 for example visual stimuli). In the method of adjustment task, the participants had to set the weight of the avatar to match their current body weight. Additionally, the participants were asked to adjust the weight of the avatar to match their ideal weight.

\subsection{Experimental Procedure}

At the very beginning of the experiment, each participant had to fill out a self-esteem questionnaire [Rosenberg 1965]. The experiment consisted of four sessions. The order of the sessions was randomized. The presentation time of the visual stimuli for all tasks was not fixed, and participants were instructed to make their estimates as accurately as possible. The only difference between the four sessions was the visual cues of the avatar (own shape with own texture; own shape with checkerboard texture; average shape with own texture; average shape with checkerboard pattern texture). Thus, in each session, the participant was presented with only one set of the personalized avatars (e.g., one row of visual stimuli from Figure 2). More specifically, this was nine avatars with $0 \%, \pm 5 \%, \pm 10 \%, \pm 15 \%$, $\pm 20 \%$ of the current participant's BMI. 
Table II. General Questionnaire at the End of the Experiment

\begin{tabular}{|l|l|}
\hline Question & \multicolumn{1}{|c|}{ Answer } \\
\hline $\begin{array}{l}\text { How much time (on average, per day) do you spend looking at your } \\
\text { body in the mirror? }\end{array}$ & $M=18.21$ minutes $(S D=20.53)$ \\
\hline Do you often think about the way you look? & Yes $(N=7)$, no $(N=6)$ \\
\hline Do you often compare the way you look with the way other people look? & Yes $(N=9)$, no $(N=4)$ \\
\hline Do you check your appearance in a mirror whenever you can? & Yes $(N=5)$, no $(N=8)$ \\
\hline Do you usually notice even small changes in your weight? & Yes $(N=3)$, no $(N=10)$ \\
\hline Are you on a weight-loss diet? & Yes $(N=2)$, no $(N=11)$ \\
\hline
\end{tabular}

Each session began with a 2AFC procedure where the participant had to answer "yes" or "no" to the following question: "Is it the same weight as you?" using joystick buttons. As soon as the participant pressed the "yes" or the "no" button, the screen went blank for 2 seconds. Then, the participant was presented with the next trial. This procedure continued for a total of 180 trials. The trials were divided into 20 blocks, in which each of the nine stimuli (e.g., one row from Figure 2) was presented once. The order of the stimuli was always randomized. After 45 trials during the 2AFC procedure, the participants had an opportunity to take a break if they needed it.

After the 2AFC procedure was complete, the participant performed a method of adjustment task, in which the participant had control over the intensity of the stimulus and her task was to adjust the weight of the avatar so that it matched her current weight. The method of adjustment task had nine trials and was programmed so that the values available for the range of the intensities of the visual stimulus were continuous (i.e., one unit of change corresponded to $0.05 \%$ BMI change). The starting point of each trial was one of the nine stimuli (in randomized order) presented in the $2 \mathrm{AFC}$ procedure.

Then, the participant performed another nine trials of the method of adjustment task (as described previously), but this time the participant was asked to adjust the weight of the avatar to what she thinks is the weight that is ideal for her. After completing the method of adjustment task for the ideal weight, participants filled out a questionnaire in which they reported (using a Likert scale) the similarity between their body and the avatar that they estimated to be the same weight as their current weight, as well as general information about the way the avatar was perceived (e.g., "How similar was the avatar to you?" or "How similar were the legs/arms/torso/face of the avatar to yours?"). The participants were specifically instructed to answer questions according to the avatar that they estimated to be the same weight as them. At the end of each session, the participant had a 5-minute break. At the very end of the experiment, participants were asked to complete a general questionnaire about their habits related to body perception and body weight (Table II).

\section{RESULTS}

\subsection{Sensitivity to Own Weight Perception: Difference for Overweight versus Underweight}

The mean proportion of the answer for "Is it the same weight as you?" is shown in Figure 3. Overall, the participants estimated the weight of the avatar with percentage of BMI (BMI\%) change of 0\%, regardless of texture or shape, to match their current weight. Yet, sensitivity to the avatars with $\pm 5 \%$, $\pm 10 \%, \pm 15 \%$, and $\pm 20 \%$ BMI change seemed to depend on whether the avatar increasingly became overweight (positive BMI\% changes) or underweight (negative BMI\% changes) as indicated by the falloff rate of the mean proportion to both sides of the $0 \%$ BMI change point. In particular, the steeper fall-off rate for positive BMI\% changes compared to negative BMI\% changes suggests that participants were somewhat more sensitive to an overweight avatar than to an underweight avatar. 

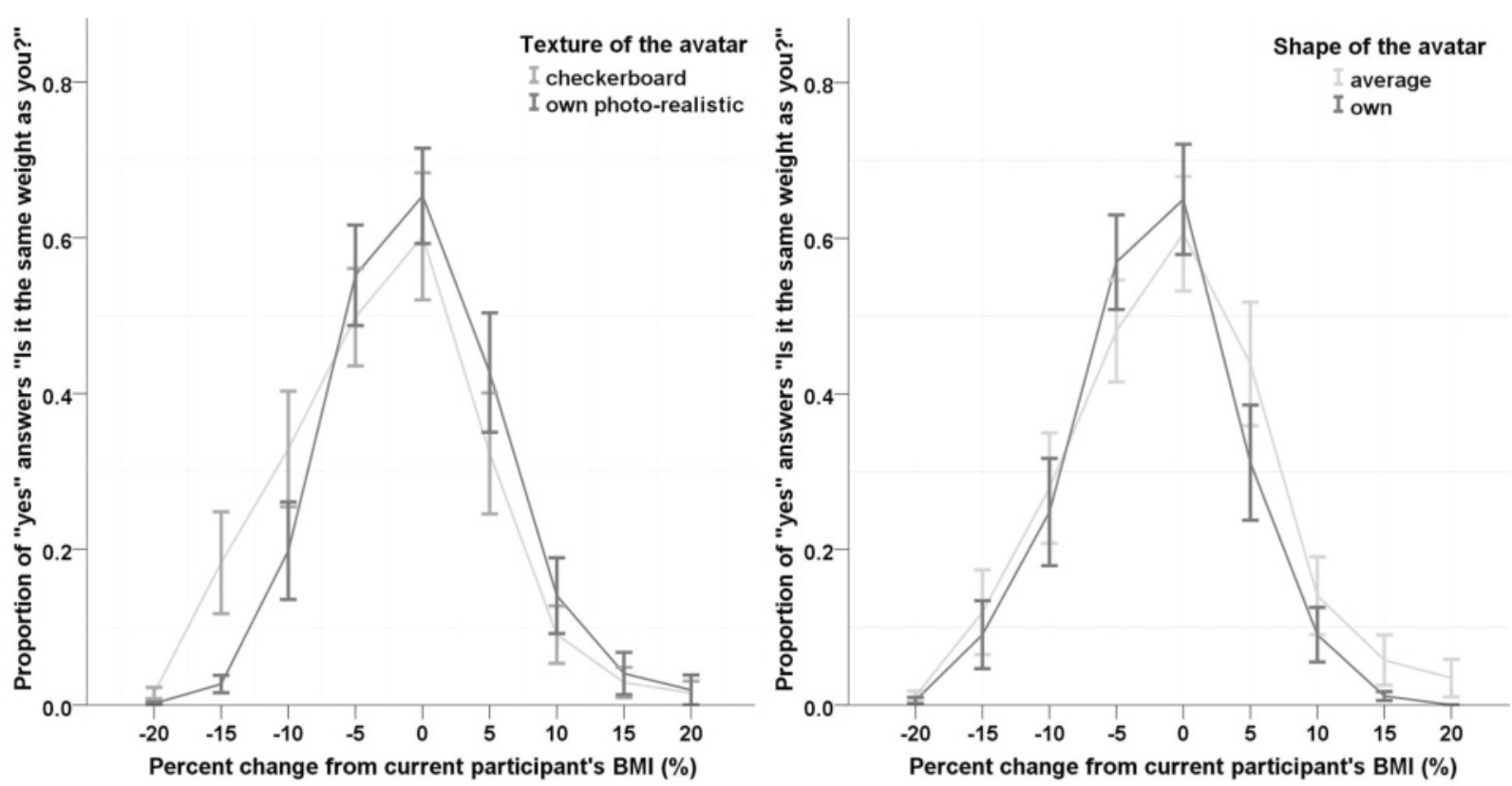

Fig. 3. The mean proportion of the answer to "Is it the same weight as you" based on the dependent variable's shape and texture and with varying amounts of BMI\% change. Error bars represent one standard error of the mean.

To statistically test these observed patterns in differences of the fall-off rate for positive compared to negative BMI\% changes, we fit psychometric functions to the mean proportions of "Is it the same weight as you?" answers separately. Specifically, we fit a cumulative Weibull function according to Wichmann and Hill [2001]. Alpha (position of the psychometric function along the $x$-axis), beta (the slope of the psychometric function), and lambda (the peak of the psychometric function) were free to vary. Gamma (flooring performance) was fixed to zero. The psychometric function was fit for each experimental condition and participant separately. The fit of the psychometric function to the mean data was good (mean $R^{2}=0.999, S E=0.0004$ ).

We examined the effect of shape and texture on lambda to examine whether manipulations of these visual cues affected the maximum proportion of "Is it the same weight as you?" answers (i.e., the height of the peak in Figure 4). A $2 \times 2$ within-subjects ANOVA showed no significant effect of shape (own vs. average), $F(1,10)=0.11, p=0.92$, or texture (own photo-realistic vs. checkerboard pattern), $F(1,10)=0.432, p=0.526$, and no interaction between shape and texture, $F(1,10)=1.223, p=$ 0.295 , on lambda (the peak of the psychometric function). Hence, the maximum response was not influenced by the visual cue manipulations.

Although there was little change with visual cues, there was a clear difference in overall slope values depending on whether the presented avatar appeared underweight or overweight compared to the participant's perceived current weight (compare left and right panel of Figure 3). To better illustrate the difference in the fall-off, the slopes are shown for the texture, shape, and BMI\% change direction (overweight vs. underweight changes) in Figure 4. Notice that higher slope values indicate greater sensitivity to BMI\% changes. We examined the effect of texture, shape, and BMI\% change direction on the slope of the psychometric function in a completely crossed within-subject ANOVA with texture (own photo-realistic vs. checkerboard pattern), shape (own vs. average), and BMI\% change direction (underweight vs. overweight) as factors and slope as the dependent variable. Because the data differed 

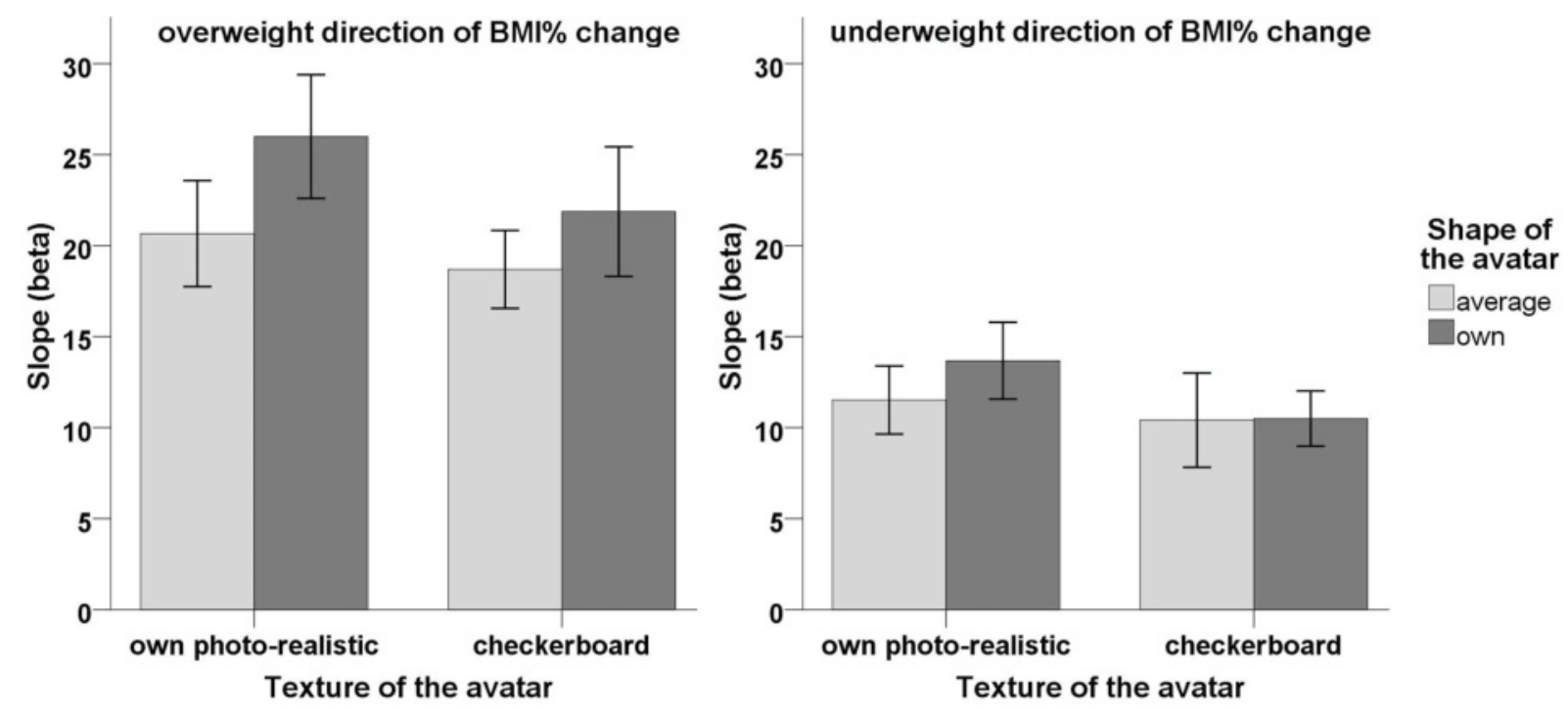

Fig. 4. The effect of shape and texture on the slope of the psychometric function (beta). Error bars represent one standard error of the mean.

from normality, we calculated the ANOVA on the power transformed slope values that restored normality. We found only a significant main effect of BMI\% change direction, $F(1,10)=17.28, p=0.002$, indicating that the slope of the overweight direction was significantly steeper than the slope of the underweight direction. The other main effects of texture and shape and two- and three-way interactions between texture, shape, and BMI\% change direction were all nonsignificant, $p>0.05$.

\subsection{The Influence of Shape (Own vs. Average) and Texture (Own Photo-Realistic vs. Checkerboard Pattern) on Weight Perception of Avatars}

Are people able to estimate their weight as seen on personalized avatars veridically? We can answer this question by considering the amount of the BMI\% change of the avatar that the participants indicated as similar to their current weight. We determined the BMI\% change values that corresponded to the maximum proportion of "avatar is my weight" answers for each participant and visual cue (shape and texture) separately. The mean values are shown in Figure 5. We investigated the effect of the visual cues on the BMI\% change values that corresponded to the maximum proportion of "avatar is my weight" by performing an ANOVA with shape (own vs. average) and texture (own photo-realistic vs. checkerboard pattern) as within-subject factors and BMI\% change (reported by the participant as their current weight) as the dependent measure. The underlying avatar's body shape had little influence (no significant effect) on the BMI\% change, $F(1,12)=0.342, p=0.57$. Interestingly, the texture of the avatar significantly influenced BMI\% change reported by the participant for their current weight estimates, $F(1,12)=14.21, p=0.003$. More specifically, participants reported that their weight was equivalent to the weight of an avatar with a significantly smaller BMI when the avatar was displayed with a checkerboard texture as compared to participants' own photo-realistic texture. The interaction between shape and texture was nonsignificant, $F(1,12)=0.103, p=0.753$.

The preceding analyses suggest that people are able to veridically portray their current weight in the four types of avatars (own shape with own texture; own shape with checkerboard texture; average shape with own texture; average shape with checkerboard pattern texture) used for this experiment 


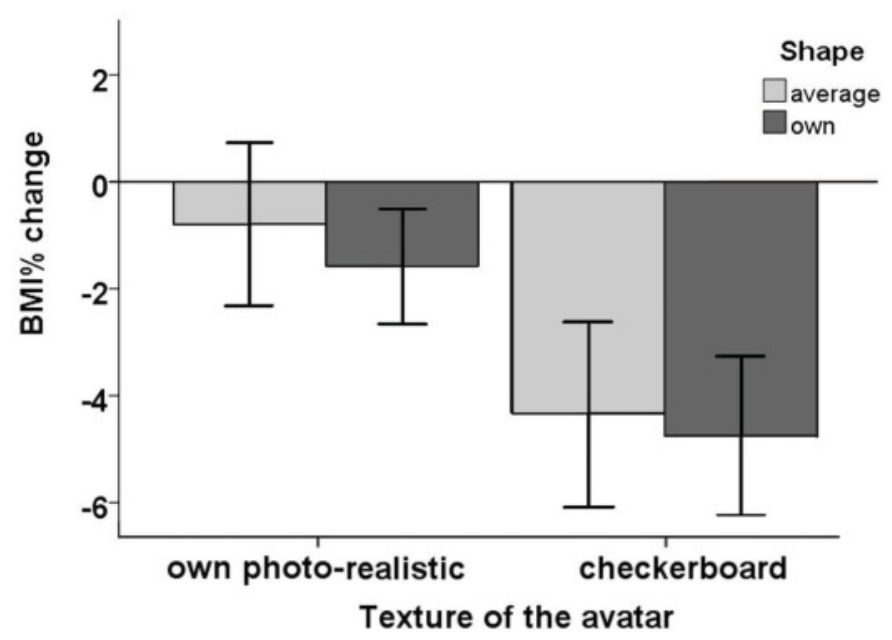

Fig. 5. The point of subjective equality (PSE) in terms of BMI\% change per experimental condition. Error bars represent one standard error of the mean.

and that the point of subjective equalities (PSEs) are all slightly smaller than the participant's current weight (see Figure 5). But also, it is important to consider that there is a tolerance range for accepting an avatar as having the same weight as oneself. The tolerance range can be determined by considering the absolute difference between the PSEs (i.e., BMI\% change that correspond to 0.5 answer proportion) on the psychometric functions fitted to the overweight and underweight data. Ranges for each texture and shape are shown in Figure 5. The data suggests that for the avatars with own photo-realistic texture and average shape, the range of the PSEs was between $-2.21 \%$ and $0.83 \%$ BMI change, with own photo-realistic texture and own shape the range was between $-0.52 \%$ and $-2.64 \%$ BMI change, with checkerboard texture and average shape the range was between $-2.63 \%$ and $-5.95 \% \mathrm{BMI}$ change, and with checkerboard texture and own shape the range was between $-3.17 \%$ and $-6.05 \% \mathrm{BMI}$ change.

\subsection{Method of Adjustment for Estimating Current Weight}

Additionally, we used the method of adjustment task for current weight (Figure 6) as a converging measure for the one-interval $2 \mathrm{AFC}$ procedure. If the method of adjustment provides consistent results with the 2AFC method, then this may be a better technique to use with the clinical population because it requires less time. Further, we used the method of adjustment task for ideal weight (see Figure 6) to determine whether there is a difference between the participants' perception of current and ideal weight. To analyze whether the visual cues (texture and shape) affected the method of adjustment task for both current and ideal weights, we ran a 2 (texture: checkerboard vs. own photo-realistic) $\times$ 2 (shape: own vs. average) $\times 2$ (task: current weight vs. ideal weight) within-subjects, repeated measures ANOVA with mean estimated weight (BMI\% change from the participant's current weight) as the dependent variable.

We found that the participants estimated their ideal weight to be significantly less than their estimated current weight, $F(1,47)=28.390, p<0.001, \eta_{p}^{2}=0.38$ (the means are shown in Figure 6). The shape and the texture, as well as the two- and three-way interactions between texture, shape, and task, were not significant-all $p s>0.16$. Although we found no significant effect of the texture on participants' estimates of current and ideal body weight, there was a trend in the estimates that was consistent with the results from the $2 \mathrm{AFC}$ procedure. Namely, to make the avatar represent the 


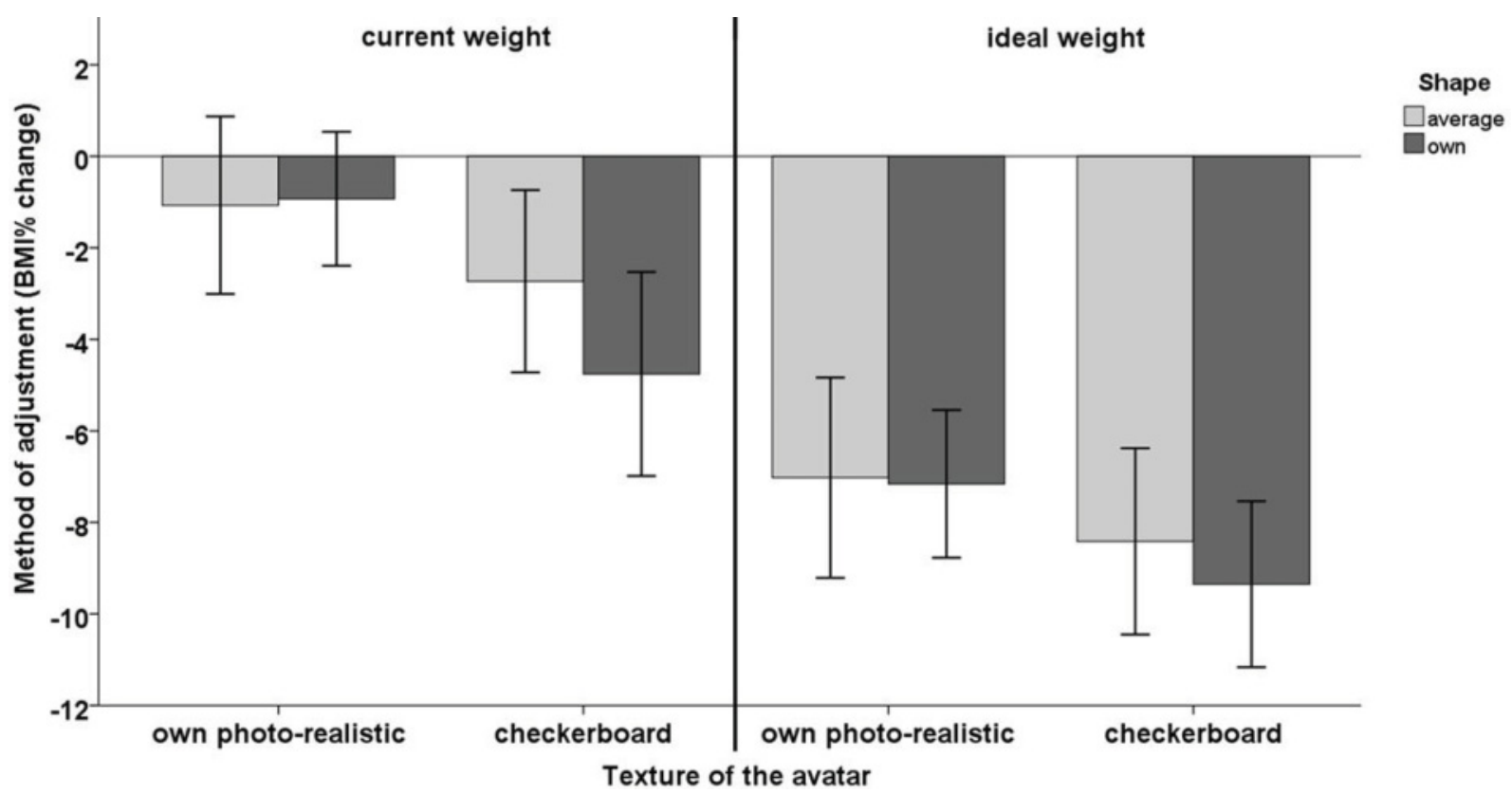

Fig. 6. The average current weight (left) and ideal weight (right) as estimated by the method of adjustment per experimental condition in terms of BMI\% change from the actual weight of the participant. Error bars represent one standard error of the mean.

requested weight (for both the ideal and current estimates), participants took more weight off the checkerboard-textured avatar than the avatar with the photo-realistic texture (compare Figure 6 to Figure 5). We also analyzed (using the same analyses as earlier but a changed dependent variable) the amount of time participants took to complete the adjustment procedure for each task and condition. We found no differences in the time taken to complete the tasks, which suggests that time was not a factor in the observed differences in adjustment across tasks and texture-all $p s>0.34$. We then investigated whether a relationship existed between the PSE-more specifically, the PSE for the positive direction (overweight avatar) and the PSE for the negative direction (underweight avatar) and the estimations from the method of adjustment task for current weight. We also found a positive correlation between the PSE for the positive direction (overweight avatar) and the estimates from the method of adjustment task for the current weight, $r(11)=0.324, p=0.032$. However, the relationship between the PSE for the negative direction (underweight avatar) and the method of adjustment estimates for the current weight was negative, $r(11)=-0.382, p=0.011$. This suggests that the method of adjustment is a possible alternative time-saving method for measuring perceived body weight in personalized avatars.

Further, for the mesh of each participant, we calculated the distance between the participant's avatar and the average avatar in the body scan database using several measurements (see Table I). First, we calculated the Mahalanobis distance between each participant's shape deformations and the average shape deformations, in terms of deformation coefficients $\beta$. Second, we estimated the uniqueness of the participant. Thus, we estimated the uniqueness of the participant compared to the average shape in terms of BMI (see height/weight distance in Table I). Finally, we also calculated the Mahalanobis distance of each participant in the distribution of 15 selected measurements spanned by the CAESAR bodies (bust-chest-circumference-under-bust, ankle-circumference, weight, 
waist-circumference-pref, shoulder-breadth, crotch-height, chest-circumference, thigh-circumference, spine-to-shoulder, hip-circumference-maximum, arm-length-shoulder-to-wrist, stature, knee-height, head-circumference, neck-base-circumference, including height and weight) (see selected measurements distance in Table I). Note that the scale of the two calculated types of distance measures varies because we used Mahalanobis distances in spaces of different dimensionality.

We then investigated the relationship between the calculated distances, the slopes, and the PSEs. All of the correlations between distances and slopes or distances and PSEs were not significant-all $p s>0.22$. The nonsignificant correlations suggest that regardless of the difference between the participant's own body and the average body, the participants were able to estimate their body weight veridically. This would suggest that for similar experiments (in which the participants are estimating own body weight), it is not necessary to use the personalized 3D scan of the participant but rather is enough to use an average avatar that has the same height as the participant.

\subsection{Considering Possible Confounds}

We tested participants' self-esteem because it is a potential confounding variable in our experiment. For calculating the self-esteem of our participants, we used the traditional Rosenberg's self-esteem scale, which ranges from 0 to 30. According to Rosenberg's self-esteem scale, scores between 15 and 25 are within the normal range, whereas scores smaller than 15 suggest low self-esteem and scores greater than 25 suggest high self-esteem. For the self-esteem questionnaire, our participants scored on average 21.75 ( $S D=5.578$, see Table I for participants' score range), with only two participants scoring below 15 and three participants scoring above 25 . We examined participants whose self-esteem deviated from normal (smaller than 15 or greater than 25) and found that their psychophysical data was not significantly different from those in the normal range-all $p s>0.356$. Thus, we do not believe that differences in self-esteem drove the observed effects, but we do concede that the sample size is probably too small to make a strong claim here. In addition, given that our sample size was 13 , we did not believe that this sample provided enough power to detect meaningful correlations between self-esteem and the observed effects (see Stuart and Ord [1994]). In the postquestionnaire, participants were asked to report their ideal weight in kilograms and reported their ideal weight to be $57.69 \mathrm{~kg}$ on average (see Table I). This is $2.99 \mathrm{~kg}$ less than their average weight. This corresponds to an average of $-4.93 \%$ BMI change.

Willingness to accept the avatar as similar to one's body could also have affected our results. The participants' ratings of the perceived similarity between their own body and the avatar that the participants estimated as similar to their current weight in each of the four sessions of the experiment are shown in Figure 7 . We analyzed the data from the similarity questions using nonparametric tests, namely Wilcoxon signed-rank tests with planned comparisons because the questionnaire data was not normally distributed. The tests indicated that the participants gave significantly lower ratings for similarity when the avatar had average shape compared to when it was the participant's exact shape, $z=-2.803, p=0.005$ (means are shown in Figure 7). The ratings for the avatar with the checkerboard pattern texture were also significantly lower compared to the ratings for the avatar with the photo-realistic texture, $z=-2.805, p=0.005$ (means are shown in Figure 7).

In addition to the similarity questions, we asked the participants whether they recognized the avatar. Their answers were as follows: avatar with own photo-realistic texture and own shape: answer "yes" $(N=13)$, answer "no" $(N=0)$; avatar with checkerboard texture and own shape: answer "yes" $(N=10)$, answer "no" $(N=3)$; avatar with own photo-realistic texture and average shape: answer "yes" $(N=4)$, answer "no" $(N=9)$; and avatar with checkerboard texture and average shape: "yes" $(N=1)$, answer "no" $(N=11)$. These results suggest that the participants were able to distinguish that the average avatar did not have their body shape. 


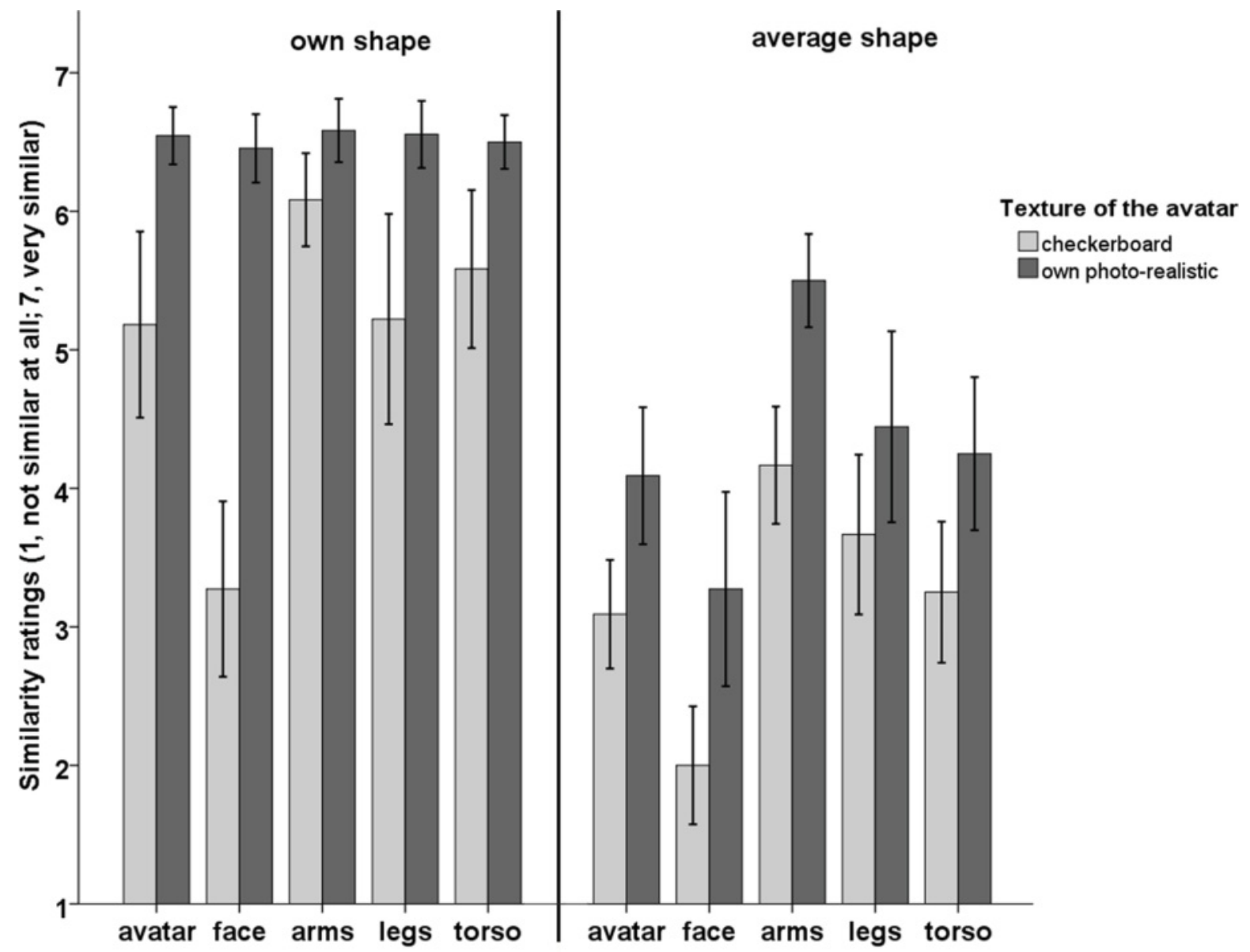

Fig. 7. The similarity ratings for avatar, face, arms, legs, and torso in the postsession questionnaires for each avatar. Error bars represent one standard error of the mean.

\section{DISCUSSION}

To our knowledge, this is the first investigation of body weight perception conducted using both the participant's photo-realistic body textures and the participant's accurate 3D body shape. Moreover, we used state-of-the-art algorithms to present participants with convincing alternative body shapes that could represent their own shape if they lose or gain a certain amount of their body weight (BMI\% change). Our results suggest that women were more willing to accept thinner avatar bodies (smaller BMI than their current BMI) as most similar to their current weight, as compared to when the avatar had a bigger body than the participant's current body. Not surprisingly, these women were less tolerant of avatar body weights that were greater than their current BMI. This willingness to accept thinner avatar bodies as one's own was pronounced in the case of avatars presented with a checkerboard texture rather than a photo-realistic one, which parallels the results of Thompson and Mikellidou [2011] on the perception of the size of mannequins that were textured with vertical or horizontal lines. To our knowledge, the research of Thompson and Mikellidou [2011] is the only prior study to our research that has examined the potential effects of visual cues, namely the influence of 
the texture cues on perceived body size. Our findings also show that the similarity of the shape of the avatar (i.e., an avatar with the participant's own body shape vs. an avatar with average shape) did not have an effect on perceiving an avatar's body weight as similar to one's own. However, this finding was not completely surprising, because even the average female avatar still had some anthropomorphic features of the participant, such as the same height, weight, and BMI. Although the results from the questionnaire show that the participants were able to distinguish that the average avatar did not have their body shape, they still veridically estimated their body weight when the avatar had average shape. Thus, our results suggest that the participants would accept an average avatar that had an equivalent BMI (i.e., height and weight) as a representation of their own weight.

Our findings showed that the participants veridically perceived their body weight when the avatar had own photo-realistic texture and either average or own body shape. These results are consistent with the findings from previous research that investigated the perception of own body size [Mischner et al. 2013] using distorted photographs of the participants' bodies. In our experiment, one reason people may have perceived their body more veridically with the photo-realistic texture is that this texture carries some lighting information (although only a small amount due to uniform lighting from all directions when captured). Thus, the photo-realistic texture provides shape from shading information that might provide additional shape cues. The clothes on the body also provide shape information. Thus, the photo-realistic texture allows one to see more local shape features to guide estimates. In contrast to the photo-realistic texture, which portrays shape-specific features such as small wrinkles and irregularities of the shape, the checkerboard texture has no directional lighting information and masks some of these features. This suggests that the quality of the texture, and the amount of information that it provides, has an influence on the belief or desires about the person's own body weight. Another potential reason for the influence of texture on weight perception of these avatars is the fact that the photo-realistic texture also provides more detailed information about face-specific features (e.g., eye color, mouth color). The texture on the face may have simply drawn the attention of the participant to the changes in the both face shape and body shape regardless of how similar the avatar was perceived to be to the participant, resulting in a greater accuracy of estimated weight.

Our results have several implications for future work. First, researchers who are interested in displaying avatars to participants may be able to use body models of female avatars with average shape that have a similar height and weight to the current participant to accurately represent a female participant's own size. Therefore, creating databases of average avatars will certainly be important for furthering work in this area. Our findings are also relevant for the development of new therapies to help patients with body image disorders. For instance, texture cues in portrayed representations of the patient could be manipulated to minimize the discrepancy between the perceived and the actual body size of patients with body image disorders. Setups similar to ours could be used to gain a better understanding of the impact of clothes on the perception of body weight, which could have commercial value. The influence of texture information on the ability to estimate shape may have been due to increased task difficulty in the case of the checkerboard texture. It would be interesting to directly manipulate task difficulty in the future to test whether such manipulations also lead to a greater bias toward lower BMIs when estimating one's own weight. Finally, using personalized avatars for assessing body perception, we can also investigate new approaches for measuring perceptual similarity.

We acknowledge that this study represents an initial step toward understanding how people perceive their body size as depicted by virtual, 3D avatars. Although promising, the observed results leave open many questions for future work. For example, if the avatar portrayed considerable changes in body dimensions as compared to the women's true body size or shape, it is possible that there would be an effect of the shape on the perception of body weight. Future work is planned to assess whether other changes to body dimension (e.g., height, shoulder width, limb length) could have more of an effect on 
the similarity of an avatar's body weight to one's own. In addition, the use of the personalized avatars in future experiments would enable us to investigate the impact of perspective or orientation (such as a rotated avatar to the side) on the perception of body weight. Moreover, using different virtual reality setups, we can investigate and compare perception of body weight on avatars shown from the firstperson perspective (e.g., using head-mounted displays) and the third-person perspective (e.g., using a large-screen display in a similar setup as ours). Further, the avatars in this study were displayed on a large, stereoscopic screen immersive display. Obviously, if we hope to use these types of scanning techniques and experimental procedures with patient populations, it would be important to replicate these findings using different types of displays that could more easily be transported to hospitals, such as mobile devices or commodity-immersive head-mounted displays. Finally, we would be remiss to not acknowledge that this work was only done with females. Males could use very different body dimensions to estimate weight. We wanted to limit these potential confounds in the current study but hope to extend our work not only to men but also women experiencing changes to their body weight during pregnancy, and those who have recently gained or lost a substantial amount of weight.

\section{CONCLUSION}

Our results showed that women are more willing to accept a thinner body (smaller BMI) as most similar to their actual body weight, as compared to when the avatar had a bigger body than the participant's current body. The shape of the avatar (matched to the participants' bodies or an average female shape with the same height and BMI) had little effect on the perception of body weight. Interestingly, the avatars with checkerboard texture needed to be significantly thinner than the avatars with photorealistic texture in order to represent the participants' current weight, suggesting that the avatars with checkerboard texture appeared bigger. The participants perceived their body weight veridically when they saw their own photo-realistic texture. Our findings have implications for developing new measures for patients with body image disorders, as well as researchers interested in understanding how the perception of avatars might influence interaction in virtual environments. Our findings also have implications for computer graphics applications that aim to create avatars of individuals (games, training applications) and suggest that women can veridically project (portray) their body weight on an average body shape that is based on body measurements (e.g., weight and height) of the participant, whereas the photo-realistic texture may be necessary to recognize the body as one's own shape.

\section{ACKNOWLEDGMENTS}

We would like to thank Naureen Mahmood for useful suggestions and discussions related to the features of the body scans; Emma-Jayne Holderness and Sophie Lupas for scanning the participants; Joachim Tesch for help with Unity programming, specifically Asset Bundles; Trevor Dodds and Aurelie Saulton for suggestions and discussions about the psychophysics experiment and perception of body size; Eric Rachlin and Jessica Purmort for early discussions; and the Alexander von Humboldt Foundation and the National Science Foundation (NSF IIS-11-16636), as well as the EC FP7 project VRHYPERSPACE (AAT-2011-RTD-1-285681).

\section{REFERENCES}

Dragomir Anguelov, Praveen Srinivasan, Daphne Koller, Sebastian Thrun, Jim Rodgers, and James Davis. 2005. SCAPE: Shape completion and animation of people. ACM Transactions on Graphics 24, 3, 408-416.

Finn Askevold. 1975. Measuring body image: Preliminary report on a new method. Psychotherapy and Psychosomatics 26, 71-77.

Federica Bogo, Javier Romero, Matthew Loper, and Michael J. Black. 2014. FAUST: Dataset and evaluation for 3D mesh registration. In Proceedings of the IEEE Conference on Computer Vision and Pattern Recognition (CVPR). 
David A. Brodie, Peter D. Slade, and H. Rose. 1989. Reliability measures in distorting body-image. Perceptual and Motor Skills $69,723-732$.

Heinrich H. Bülthoff and Shimon Edelman. 1992. Psychophysical support for a two-dimensional view interpolation theory of object recognition. In Proceedings of the National Academy Science of the United States of America. 89, 1, 60-64.

Thomas F. Cash, Diane W. Cash, and Jonathan W. Butters. 1983. Mirror, mirror, on the wall: Contrast effects and self-evaluations of physical attractiveness. Personality and Social Psychology Bulletin 9, 3, 351-358.

Thomas F. Cash and Edwin A. Deagle. 1997. The nature and extent of body-image disturbances in anorexia nervosa and bulimia nervosa: A meta-analysis. International Journal of Eating Disorders 22, 107-125.

Frederique De Vignemont. 2011. Embodiment, ownership and disownership. Consciousness and Cognition 20, 1, 81-93.

Jessica Doolen, Patricia T. Alpert, and Sally K. Miller. 2009. Parental disconnect between perceived and actual weight status of children: A metasynthesis of the current research. Journal of the American Academy of Nurse Practitioners 21, 3, 160-166.

April Fallon and Paul Rozin. 1985. Sex differences in perceptions of desirable body shape. Journal of Abnormal Psychology 94, $1,102$.

Clare Farrell, Michelle Lee, and Roz Shafran. 2005. Assessment of body size estimation: A review. European Eating Disorders Review 13, 2, 75-88.

Richard J. Freeman, Cheryl D. Thomas, Leslie Solyom, and Michael A. Hunter. 1984. A modified video camera for measuring body image distortion: Technical description and reliability. Psychological Medicine 14, 411-416.

Christina T. Fuentes, Matthew R. Longo, and Patrick Haggard. 2013. Body image distortions in healthy adults. Acta Psychologica 144, 2, 344-351. DOI : http://dx.doi.org/10.1016/j.actpsy.2013.06.012

Myron L. Glucksman and Hirsch Jules. 1968. The response of obese patients to weight reduction: A clinical evaluation of behavior. Psychosomatic Medicine 30, 1-11.

Sarah Grogan. 2007. Body Image: Understanding Body Dissatisfaction in Men, Women, and Children (2nd ed.). Routledge.

Teruo Hashimoto and Atsushi Iriki. 2013. Dissociations between the horizontal and dorsoventral axes in body-size perception. European Journal of Neuroscience 37, 11, 1747-1753.

David Hirshberg, Matthew Loper, Eric Rachlin, and Michael J. Black. 2012. Coregistration: Simultaneous alignment and modeling of articulated 3D shape. In Proceedings of the European Conference on Computer Vision (ECCV). Lecture Notes in Computer Science, Vol. 7577. Springer-Verlag, 242-255.

Matthew R. Longo and Patrick Haggard. 2012a. Implicit body representations and the conscious body image. Acta Psychologica $141,2,164-168$.

Matthew R. Longo and Patrick Haggard. 2012b. What is it like to have a body? Current Directions in Psychological Science 21, 2, 140-145.

Randi E. McCabe, Traci McFarlane, Janet Polivy, and Marion P. Olmsted. 2001. Eating disorders, dieting, and the accuracy of self-reported weight. International Journal of Eating Disorders 29, 1, 59-64.

Isabelle H. S. Mischner, Hein T. van Schie, Danil H. J. Wigboldus, Rick B. van Baaren, and Rutger C. M. E. Engels. 2013. Thinking big: The effect of sexually objectifying music videos on bodily self-perception in young women. Body Image 10, 1 , 26-34.

Perceiving Systems MPI IS. 2011. Body Visualizer. MPI IS Perceiving Systems Department, Copyright Max Planck Gesellschaft. Retrieved July 2, 2014, from http://bodyvisualizer.com.

Ivelina V. Piryankova, Hong Yu Wong, Sally Linkenauger, Catherine Stinson, Matthew Longo, Heinrich H. Bülthoff, and Betty J. Mohler. 2010. Owning an overweight or underweight body: Distinguishing the physical, the experienced and the virtual body. PLOS ONE (accepted).

Giuseppe Riva. 2010. Neuroscience and Eating Disorders: The Role of the Medial Temporal Lobe. Retrieved July 21, 2014, from http://hdl.handle.net/10101/npre.2010.4235.1

Giuseppe Riva. 2011. The key to unlocking the virtual body: Virtual reality in the treatment of obesity and eating disorders. Journal of Diabetes Science and Technology, 283-292.

Kathleen M. Robinette and Hein Daanen. 1999. The CAESAR Project: A 3-D surface anthropometry survey. In Proceedings of the Second International Conference on 3-D Digital Imaging and Modeling. 380-386.

Morris Rosenberg. 1965. Society and the Adolescent Self-Image. Princeton University Press, Princeton, NJ.

Paul Schilder. 1935. The Image and Appearance of the Human Body: Studies in the Constructive Energies of the Psyche. International University Press, New York, NY.

Andrew J. Schofield, Peng Sun, and Giacomo Mazzilli. 2013. Shape Perception in Human and Computer Vision: An Interdisciplinary Perspective. Springer-Verlag, London. 
Peter D. Slade and Gregory F. Russell. 1973. Awareness of body dimensions in anorexia nervosa: Cross-sectional and longitudinal studies. Psychological Medicine 3, 188-199.

Monique A. M. Smeets, Filip Smit, Geert E. M. Panhuysen, and J. David Ingleby. 1997. The influence of methodological differences on the outcome of body size estimation studies in anorexia nervosa. British Journal of Clinical Psychology 36, $263-277$.

Alan Stuart and Keith Ord. 1994. Kendall's Advanced Theory of Statistics (6th ed.). Edward Arnold, London.

Albert J. Stunckard, Thorkild Sorensen, and Fini Schulsinger. 1983. The Genetics of Neurological and Psychiatric Disorders. Raven Press, New York, NY.

Peter Thompson and Kyriaki Mikellidou. 2011. Applying the Helmholtz illusion to fashion: Horizontal stripes won't make you look fatter. $i$-Perception 2, 1, 69-76.

Arthur C. Traub and J. J. Orbach. 1964. Psychophysical studies of body-image: I. The adjustable body-distorting mirror. Archives of General Psychiatry 11, 1, 53-66.

Christian Wallraven, Adrian Schwaninger, Sandra Schuhmacher, and Heinrich H. Bülthoff. 2002. View-based recognition of faces in man and machine: Re-visiting inter-extra-ortho. In Biologically Motivated Computer Vision, Heinrich H. Bülthoff, Christian Wallraven, Seong-Whan Lee, and Tomaso A. Poggio (Eds.). Lecture Notes in Computer Science, Vol. 2525. Springer, Berlin, Heidelberg, 651-660.

Alexander Weiss, David Hirshberg, and Michael J. Black. 2011. Home 3D body scans from noisy image and range data. In Proceedings of the International Conference on Computer Vision (ICCV). IEEE, Los Alamitos, CA, 1951-1958.

Felix A. Wichmann and N. Jeremy Hill. 2001. The psychometric function: I. Fitting, sampling and goodness-of-fit. Perception and Psychophysics 63, 8, 1293-1313.

Peter Willemsen, Amy A. Gooch, William B. Thompson, and Sarah H. Creem-Regehr. 2008. Effects of stereo viewing conditions on distance perception in virtual environments. Presence: Teleoperators and Virtual Environments 17, 1, 91-101.

Received June 2014; revised July 2014; accepted July 2014 